\title{
Antioxidants and cancer: a debate on prevention, progression, hormesis, and cruciferous vegetables
}

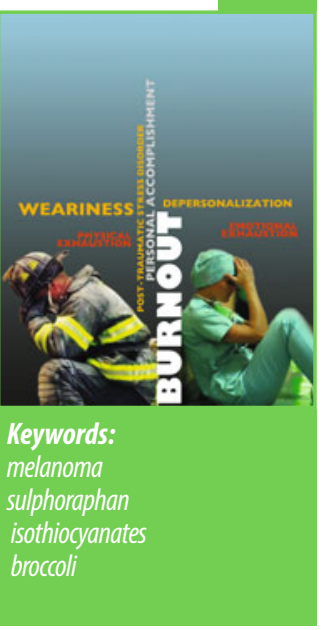

(C) Springer - CEC Editore 2015

Antioxidants should maintain cellular health. That is why millions of people consume supplements containing vitamin $\mathrm{E}, \beta$-carotene, or a host of phytochemicals every day. But now two new studies suggest, adding to a growing body of research, that antioxidant supplements actually could have a harmful effect for the prevention of cancer.

The first study [1], carried out in mice, shows that some antioxidants may so modify malignant melanoma cells - the most aggressive types of skin cancer - to promote their dissemination to different parts of the body. Such a progression makes the disease even deadlier. As described by the Authors, administration of $\mathrm{N}$-acetylcysteine (NAC) increases lymph node metastases without having an impact on the number and size of primary tumors. Similarly, NAC and a soluble vitamin E analog (Trolox) markedly increased the migration and invasive properties of human malignant melanoma cells without affecting their proliferation. Both antioxidants increased the ratio between reduced and oxidized glutathione in melanoma cells and in lymph node metastases, and the increased migration relied on new glutathione synthesis. Moreover, both NAC and Trolox increased the activation of a protein, called RHOA, which normally helps cells to spread all over the body, and blocking downstream RHOA signaling abolished antioxidant-induced migration. These results suggest that antioxidants, along with the glutathione system, play a previously unappreciated role in malignant melanoma progression. Of course, mice are not humans, but these results deserve to be taken into consideration.

The second study [2] presents the results of a double-blind randomized, placebo-controlled trial concerning the clinical activity of a dietary supplement containing a combination of lycopene (35 mg), selenium $(55 \mu \mathrm{g})$, and $600 \mathrm{mg}$ of green tea catechins (GTCs) in men with multifocal high grade prostatic intraepithelial neoplasia (mHGPIN) and/or atypical small acinar proliferation (ASAP). In this study, performed between 2009 and 2014, 60 patients with primary mHGPIN and/or ASAP were enrolled and treated daily for 6 months. At month 6, 53 men underwent re-biopsy and 13 of them $(24.5 \%)$ were diagnosed with prostate cancer. Ten of the 13 belonged to the treated group and three to the placebo group. The difference was not significant but was close to significance $(p=0.053)$. Even though three additional prostate carcinomas were found in the placebo group at follow-up (37 months), reducing further the very poor statistical difference observed between the two groups, the tendency shown by the results remains worrying. According to the Authors' conclusions, administration of high doses of lycopene, selenium, and GTCs in men harboring HGPIN and/or ASAP was associated with a higher incidence of prostate can- 
cer at re-biopsy and therefore "the use of these supplements should be avoided".

We must, however, admit that even in the past the role played by possible antioxidant therapies aimed at preventing cancer has been disputed. Previous research on the use of supplements with antioxidants had led to the hypothesis of a cancer promoting effect. In 1994 a large study [3] concluded that massive daily doses of $\beta$-carotene increased the risk of lung cancer in male smokers by $18 \%$ and a clinical trial [4] carried out in 1996 was prematurely stopped after researchers discovered that high doses of $\beta$-carotene and vitamin A increased the risk of lung cancer by $28 \%$ in smokers and workers exposed to asbestos. More recently, a study published in 2011 [5] involving more than 35,500 men over 50 years of age, found that high doses of vitamin $\mathrm{E}$ led to an increase (17\%) in the risk of prostate cancer.

These findings have puzzled many researchers because it is widely believed that antioxidants should decrease the risk of cancer by neutralizing free radicals that damage cells and cause cancer. Which assumptions might explain this apparent paradox? A first hypothesis could be that antioxidants, taken in high enough doses, could protect from free radicals but favor the spread of tumor cells. Indeed some data suggest that antioxidants may be as useful to cancer cells as they are to normal cells. In the study performed in mice, for instance, the Authors decided to study melanoma both because the incidence rates are increasing and because it is known that this cancer could be sensitive to the effects of free radicals. So the scientists administered the antioxidant NAC in mice that had been genetically engineered to be susceptible to melanoma. The dose per kilogram of weight given to the mice was similar to the amount people generally consume with supplements. Although the treated mice did not develop more skin cancers than mice not fed antioxidants, they were disproportionately affected by double lymph node cancer, a clear sign of cancer spread (metastasis). When the researchers added the NAC or a form of vitamin $\mathrm{E}$ to human melanoma cells in culture, there was confirmation of the fact that antioxidants improved the ability of cells to move and invade neighboring tissues. Could it be that antioxidants strengthen the protection of cancer cells? In the metastatic tumor cells of treated mice, the Authors found higher levels of glutathione than in untreated mice. The treated mice also had a higher ratio between glutathione and glutathione disulfide, the latter being the transformed molecule after it has neutralized free radicals [1]. These results suggest that when the body consumes an excess of antioxidants, its tumor cells have more antioxidants than they themselves have produced. The cancer cells could store the surplus, improving their ability to protect themselves. This idea is also supported by a study [6] showing that some genes that drive cancer growth activate other genes that produce intrinsic antioxidants.

Antioxidant supplementation may help cancer cells in other ways also. The recent research performed in mice has concluded that glutathione affects the activity of RHOA, which helps cancer cells to spread. Indeed the Authors confirmed that the surplus glutathione in treated mice caused an increase in the levels of RHOA in metastatic cells [1]. In a recent study on lung cancer [7], the Authors also found that in lung cancer cells antioxidant supplements caused the decommissioning of a wellknown tumor suppressor gene called p53. It is believed that its inactivation is critical for promoting the metastatic process. Another work [8] has shown that antioxidants help the cells of breast cancer to survive when detached from the extracellular matrix, the network of protein surrounding the cells. These molecular studies could clarify the results of the large human studies that have investigated the involvement of antioxidants in prostate cancer development [2]. Is it possible that antioxidant supplements, rather than triggering cancer, could accelerate the progression of pre-existing undiagnosed tumors, making the subsequent discovery of the 
disease likely? In other words, is it possible that antioxidants could prevent DNA damage and thereby prevent the onset of cancer, but once the cancer has started, could facilitate the malignant behavior of cancer cells?

A second explanation could be that we mess things up when we classify as 'antioxidants' very different types of molecules which surely have many others properties. We all agree that NAC, vitamin E, selenium, curcumin, and GTC are 'antioxidants', but they are also something else. And could it be that this 'something else' is responsible for the cancer promoting effects observed sometimes? This would mean that the fact that it is simply an antioxidant is not the real 'problem' of a substance. Being an antioxidant could be a simple epiphenomenon and the observed cancer promoting effects could be due to still unknown properties of some of the molecules that we simply call 'antioxidants'. This would also mean that some, or even most, of the 'antioxidants' do not have any cancer promoting effect at all. Needless to say, the scientific literature also reports totally opposite results, where for instance antioxidant therapies prevent, or should prevent, cancer [9-11].

There is a third hypothesis based on a very important topic. Too often it is believed that 'antioxidizing' is always a good thing. This could be untrue. If, as already said, some studies examining highdose vitamin $\mathrm{E}$ have shown some tumor promoting effects and not a reduction, physical activity and sport (which oppositely generate many free radicals) or crucifers (whose isothiocyanates are surely pro-oxidants) protect against cancers and cardiovascular accidents [12-19].

It must be remembered that the body produces its own antioxidants (glutathione, catalase, peroxidase, etc.), but if we replace them with excessive exogenous supplementation, the body reduces its own production. This is an adaptive negative feedback response. For instance, the relationship between physical exercise and oxidative stress is extremely complex, and depends on the mode, intensity, and duration of exercise. Physical activity leads to increased oxidative stress, but this increase appears to be beneficial for health. This same stimulus is in fact necessary to allow up-regulation of endogenous antioxidant defenses, a phenomenon known as hormesis. Supporting endogenous defenses with additional oral antioxidant supplementation may be a suitable non-invasive tool for preventing or reducing excessive oxidative stress during acute and severe training. However, an excess of exogenous antioxidants may have detrimental effects on health and performance by blocking the hormetic process [20]. Similarly to physical activity (and also caloric restriction, which is not discussed here), isothiocyanates from cruciferous vegetables act to promote a constant low level oxidative stress able to support the hormetic feedback and therefore leading to an endogenous increase in antioxidant defenses [21]. The pro-oxidant role played by isothiocyanates activates Nrf2 (a transcription nuclear factor), which along with the protein MAF binds DNA activating transcription of the ARE (antioxidant response elements) proteins. The up-regulation of the ARE system stimulates a relevant increase in the cellular antioxidant defenses [22].

How much were the antioxidant responses reduced by 6 months of therapy with antioxidant supplementation [2]? And once suspended, by much were endogenous antioxidants reduced in the treated subjects? Of course we do not have these or other answers. However, from an evolutionary perspective we do know that we have developed "the feedbacks". In response to most of our actions healthy eukaryotic cells respond in adaptive ways. If we administer hormones to a healthy human body, it responds by reducing its own production. Similarly, if we administer meaningless excessive doses of antioxidants, healthy cells tend to reduce antioxidant endogenous production. On the contrary, we should exploit the hormetic mechanisms stimulating new endogenous antioxidant responses. Indeed, epidemiology suggests that a cruciferous vegetable- 
based diet is really protective in terms of cancer [23], clinical trials with a cruciferous vegetablebased therapy clearly show a protective effect [24], and isothiocyanates administration triggers a strong and effective anticancer/antioxidant response [25]. A lifelong diet based on cruciferous vegetables presents some problems (isothiocyanates are sulphur compounds and not particularly liked; moreover cooking destroys most of the myrosinase, the enzyme responsible for converting the inactive glucosinolates into the active isothiocyanates $[26,27]$ ) but a prevention therapy based on the administration of cruciferous extracts, especially if characterized by the presence of all the different types of protective isothiocyanates, could confer a real protective effect. There are more than 120 isothiocyanates which are classified as aliphatic, aryl, arilaliphatic, and indoles isothiocyanates and a procedure taking into consideration their different solvent solubility would provide a way to extract them with good performance. A cruciferous vegetable-based, pro-oxidant supplement enriched with the various type of isothiocyanates so to resemble as much as possible the molecular bouquet of the corresponding vegetables, could be a possible right choice.

In conclusion, recent papers seem to confirm what has already been occasionally observed in the past: antioxidant supplements intended to be cancer protective appear to be cancer promoting. Consequently in some cases, the results are worrying, especially with botanical supplements like GTC, although the clear chemical characteristics of the tested products along with their possible pharmacokinetic and/or pharmacodynamic properties are not reported in the studies.

However, it could be simply that, just as cytotoxic chemotherapy or radiotherapy used to halt cancer can also provoke it, antioxidant therapy, intended to antagonize free radicals and then cancer development, could actually also help cancer cells to survive.

Another issue to consider is the classification of a phytochemical as an antioxidant. Most of the time, antioxidant properties are just one facet of a complex biological profile. Therefore, the real mechanism of the cancer promoting effects might be due to another element of this profile which has nothing to do with antioxidant activity. If so, we should go deeper into the research to determine which antioxidant molecules, apart from being antioxidants, are also cancer promoting agents.

Last but not least, it could be that, to a large extent, the role played by antioxidants is counter to the protective role exerted by the hormetic mechanisms. In this sense a protective effect could be obtained by pro-oxidant molecules like isothiocyanates. Indeed, the scientific literature substantially agrees in considering that the pro-oxidant cruciferous vegetables, that behave as veritable "prooxidant, hormesis-exploiting, cancer preventive, herbal drugs", have a relevant protective role.

\section{Conflict of interest}

Francesco Di Pierro is owner of Velleja Research.

\section{References}

1. Le Gal K, Ibrahim MX, Wiel C, Sayin VI, Akula MK, Karlsson C, Dalin MG, Akyürek LM, Lindahl P, Nilsson J, Bergo MO (2015) Antioxidants can increase melanoma metastasis in mice. Sci Transl Med 7(308):308re8

2. Gontero P, Marra G, Soria F, Oderda M, Zitella A, Baratta F, Chiorino G, Gregnanin I, Daniele L, Cattel L, Frea B, Brusa P (2015) A randomized double-blind placebo controlled phase I-II study on clinical and molecular effects of dietary supplements in men with precancerous prostatic lesions. Chemoprevention or "chemopromotion"? Prostate 75(11): 1177-1186

3. [No authors listed] (1994) The effect of vitamin E and beta carotene on the incidence of lung cancer and other cancers in male smokers. The Alpha-Tocopherol, Beta Carotene Cancer Prevention Study Group. N Engl J Med 330(15): 1029-1035

4. Omenn GS, Goodman GE, Thornquist MD, Balmes J, Cullen MR, Glass A, Keogh JP, Meyskens FL, Valanis B, Williams JH, Barnhart S, Hammar S (1996) Effects of a combination of beta carotene and vitamin A on lung cancer and cardiovascular disease. N Engl J Med 334(18):1150-1155

5. Klein EA, Thompson IM Jr, Tangen CM, Crowley JJ, Lucia MS, Goodman PJ, Minasian LM, Ford LG, Parnes HL, Gaziano JM, Karp DD, Lieber MM, Walther PJ, Klotz L, Par- 
sons JK, Chin JL, Darke AK, Lippman SM, Goodman GE, Meyskens FL Jr, Baker LH (2011) Vitamin E and the risk of prostate cancer: the Selenium and Vitamin E Cancer Prevention Trial (SELECT). JAMA 306(14):1549-1556

6. De Nicola GM, Karreth FA, Humpton TJ, Gopinathan A, Wei C, Frese K, Mangal D, Yu KH, Yeo CJ, Calhoun ES, Scrimieri F, Winter JM, Hruban RH, Iacobuzio-Donahue C, Kern SE, Blair IA, Tuveson DA (2011) Oncogene-induced Nrf2 transcription promotes ROS detoxification and tumorigenesis. Nature 475(7354):106-109

7. Mendelsohn AR, Larrick JW (2014) Paradoxical effects of antioxidants on cancer. Rejuvenation Res 17(3):306-311

8. Davison CA, Durbin SM, Thau MR, Zellmer VR, Chapman SE, Diener J, Wathen C, Leevy WM, Schafer ZT (2013) Antioxidant enzymes mediate survival of breast cancer cells deprived of extracellular matrix. Cancer Res 73(12):3704-3715

9. Nishino H, Tokuda H, Satomi Y, Masuda M, Osaka Y, Yogosawa S, Wada S, Mou XY, Takayasu J, Murakoshi M, Jinnno K, Yano M (2004) Cancer prevention by antioxidants. Biofactors 22(1-4):57-61

10. Nishino H, Tokuda H, Murakoshi M, Satomi Y, Masuda M, Onozuka M, Yamaguchi S, Takayasu J, Tsuruta J, Okuda M (2000) Cancer prevention by natural carotenoids. Biofactors 13(1-4):89-94

11. Epstein KR (2003) The role of carotenoids on the risk of lung cancer. Semin Oncol 30(1):86-93

12. Kushi LH, Doyle C, McCullough M, Rock CL, Demark-Wahnefried W, Bandera EV, Gapstur S, Patel AV, Andrews K, Gansler T (2012) American Cancer Society Guidelines on nutrition and physical activity for cancer prevention: reducing the risk of cancer with healthy food choices and physical activity. CA Cancer J Clin 62(1):30-67

13. Lemanne D, Cassileth B, Gubili J (2013) The role of physical activity in cancer prevention, treatment, recovery, and survivorship. Oncology (Williston Park) 27(6):580-585

14. Hupin D, Roche F, Gremeaux V, Chatard JC, Oriol M, Gaspoz JM, Barthélémy JC, Edouard P (2015) Even a lowdose of moderate-to-vigorous physical activity reduces mortality by $22 \%$ in adults aged $\geq 60$ years: a systematic review and meta-analysis. Br J Sports Med 49(19):1262-1267

15. Fisher-Wellman K, Bell HK, Bloomer RJ (2009) Oxidative stress and antioxidant defense mechanisms linked to exercise during cardiopulmonary and metabolic disorders. Oxid Med Cell Longev 2(1):43-51
16. Bai Y, Wang X, Zhao S, Ma C, Cui J, Zheng Y (2015) Sulforaphane protects against cardiovascular disease via Nrf2 activation. Oxid Med Cell Longev 2015:407580

17. Zhang Y (2010) Allyl isothiocyanate as a cancer chemopreventive phytochemical. Mol Nutr Food Res 54(1):127-135

18. Wang D, Upadhyaya B, Liu Y, Knudsen D, Dey M (2014) Phenethyl isothiocyanate upregulates death receptors 4 and 5 and inhibits proliferation in human cancer stemlike cells. BMC Cancer 14:591

19. Kumar G, Tuli HS, Mittal S, Shandilya JK, Tiwari A, Sandhu SS (2015) Isothiocyanates: a class of bioactive metabolites with chemopreventive potential. Tumour Biol 36(6):40054016

20. Pingitore A, Lima GP, Mastorci F, Quinones A, Iervasi G, Vassalle C (2015) Exercise and oxidative stress: potential effects of antioxidant dietary strategies in sports. Nutrition 31(7-8):916-922

21. Pall ML, Levine S (2015) Nrf2, a master regulator of detoxification and also antioxidant, anti-inflammatory and other cytoprotective mechanisms, is raised by health promoting factors. Sheng Li Xue Bao 67(1):1-18

22. Nakamura Y, Miyoshi N (2010) Electrophiles in foods: the current status of isothiocyanates and their chemical biology. Biosci Biotechnol Biochem 74(2):242-255

23. Tse G, Eslick GD (2014) Cruciferous vegetables and risk of colorectal neoplasms: a systematic review and meta-analysis. Nutr Cancer 66(1):128-139

24. Alumkal JJ, Slottke R, Schwartzman J, Cherala G, Munar M, Graff JN, Beer TM, Ryan CW, Koop DR, Gibbs A, Gao L, Flamiatos JF, Tucker E, Kleinschmidt R, Mori M (2015) A phase II study of sulforaphane-rich broccoli sprout extracts in men with recurrent prostate cancer. Invest New Drugs 33(2):480-489

25. Zhang Y, Talalay P, Cho CG, Posner GH (1992) A major inducer of anticarcinogenic protective enzymes from broccoli: isolation and elucidation of structure. Proc Natl Acad Sci U S A 89(6):2399-2403

26. Ghawi SK, Shen Y, Niranjan K, Methven L (2014) Consumer acceptability and sensory profile of cooked broccoli with mustard seeds added to improve chemoprotective properties. J Food Sci 79(9):S1756-S1762

27. Dosz EB, Jeffery EH (2013) Modifying the processing and handling of frozen broccoli for increased sulforaphane formation. J Food Sci 78(9):H1459-H1463 